\title{
Prevalência das alterações eletrocardiográficas nos pacientes da Rede de Teleassistência de Minas Gerais, MG, Brasil
}

\section{Prevalence of Electrocardiographic Changes in Patients of Teleassistance Network of Minas Gerais, Minas Gerais, Brazil}

Franciele Guimarães de Brito ${ }^{1}$ Aurélia Aparecida de Araújo Rodrigues ${ }^{2}$ Elmiro Santos Resende ${ }^{3}$ João Batista Destro Filho ${ }^{4}$ Rodolfo dos Santos Ribeiro ${ }^{5}$

\footnotetext{
1. Enfermeira. Doutoranda em Engenharia Elétrica. Universidade Federal de Uberlândia (UFU) / Departamento de Pós-graduação da Faculdade de Engenharia Elétrica. E-mail: francieleguimaraes@hotmail.com. Autor correspondente.

2. Matemática. Doutora em Engenharia de Produção. Professora Associada da Universidade Federal de Uberlândia (UFU) / Faculdade de Matemática. E-mail: estatistica.ufu@gmail.com.

3. Cardiologista. Doutor em Medicina. Professor Associado da Universidade Federal de Uberlândia (UFU) / Faculdade de Medicina. E-mail: esr_udi@hotmail.com.

4. Engenheiro Eletricista. Doutor em Processamento de Sinais. Professor Adjunto da Universidade Federal de Uberlândia (UFU) / Departamento de Pósgraduação da Faculdade de Engenharia Elétrica. E-mail: jbdestrof@yahoo.com.

5. Engenheiro Biomédico. Mestre em Engenharia Biomédica. Universidade Federal de Uberlândia (UFU). E-mail: rodolfosant@gmail.com.
} 


\section{Resumo}

O eletrocardiograma é uma ferramenta muito utilizada para rastrear e detectar doenças cardíacas, que são uma das principais causas de morte no Brasil. Alguns países vêm utilizando os recursos da telemedicina para possibilitar cuidados à saúde nas situações em que a distância é um fator crítico, até mesmo no caso de doenças cardiovasculares. O Estado de Minas Gerais, por exemplo, conta desde 2006 com um sistema de telecardiologia, na Rede de Teleassistência de Minas Gerais, que já abrange mais de $90 \%$ da população mineira. O presente trabalho busca identificar as principais alterações eletrocardiográficas em pacientes da Rede de Teleassistência de Minas Gerais. Foi realizado um estudo retrospectivo, exploratório e descritivo dos eletrocardiogramas no período de janeiro a dezembro de 2011, a amostra aleatória composta por 4.000 exames, em relação aos 318.387 exames laudados nesta base de dados em 2011. Esta amostra também é estatisticamente proporcional à soma da população de todos os municípios que estão diretamente relacionados a cada polo. Todos os exames desta amostra que possuíam alterações eletrocardiográficas foram agrupados e analisados quantitativamente. A principal alteração eletrocardiográfica encontrada em todos os polos da Rede de Teleassistência de Minas Gerais foi relacionada aos bloqueios intraventriculares (25,64\%), seguido das arritmias cardíacas (22,86\%). Dessa forma, os resultados do estudo poderão fornecer subsídios que auxiliem no planejamento de políticas públicas, colaborando para nortear programas que promovam uma assistência de qualidade na área.

Palavras-chave: Eletrocardiografia; Telemedicina; Doenças Cardiovasculares.

\section{Abstract}

The electrocardiogram is a tool widely used to track and detect heart disease, which are a major cause of death in Brazil. Some countries are using the capabilities of telemedicine to provide health care in situations where the distance is a critical factor, even in the case of cardiovascular diseases. The State of Minas Gerais, for example, account since 2006 with a telecardiology system, Alarm network of Minas Gerais, which already covers more than $90 \%$ of the population. This paper seeks to identify the main ECG changes in patients of Teleassistance Network of Minas Gerais. Were conducted a retrospective study, exploratory and descriptive of the electrocardiograms in the period from January to December 2011 , the random sample consisting of 4,000 tests in relation to 318,387 exams with investigative reports in this database in 2011. This sample is also statistically proportional to the sum of the population of all municipalities that is directly related to each pole. All the tests in this sample who had ECG changes were grouped and analyzed in a quantitatively way. The primary electrocardiographic change found in all poles of the Alarm network of Minas Gerais was related to intraventricular locks (25.64\%), followed by the cardiac arrhythmias (22.86\%). In this way, the study results may provide subsidies to aid in the planning of public policy, helping to guide programs that promote a quality assistance in the area. Keywords: Electrocardiography; Telemedicine; Cardiovascular Diseases. 


\section{Introdução}

O eletrocardiograma (ECG) é o registro da atividade elétrica cardíaca na superfície do tórax ${ }^{(1)}$. É amplamente utilizado para rastrear e detectar doenças cardíacas, sendo uma ferramenta de baixo custo $^{(2)}$. O exame é considerado padrão ouro para o diagnóstico não invasivo das arritmias e distúrbios de condução, além de ser muito importante nos quadros isquêmicos coronarianos, constituindo-se em um marcador de doença do coração(1).

De acordo com a Organização Mundial de Saúde (OMS), as doenças isquêmicas do coração e o acidente vascular cerebral foram responsáveis por 15 milhões de mortes em 2015. Desses, 8,76 milhões de pessoas morreram por doenças isquêmicas do coração( ${ }^{(3)}$. No Brasil, as doenças do aparelho circulatório também representam as principais causas de óbitos, correspondendo a $28,6 \%$ de todas as causas de mortalidade no ano $2015^{(4)}$.

Diante desse contexto e do impacto significativo das doenças cardiovasculares no orçamento da Saúde, principalmente na atenção da alta complexidade, vários países têm utilizado os serviços de telessaúde como ferramenta de auxílio no diagnóstico e tratamento de diversas patologias ${ }^{(5,6)}$, inclusive no caso das doenças cardiovasculares ${ }^{(7)}$. O diagnóstico de doenças crônicas em adultos jovens é de extrema importância para o bom prognóstico e a não descompensação cardiovascular ${ }^{(8)}$.

Em meados de 1960, a National Aeronautics and Space Administration (NASA) fez grandes avanços na área da telemedicina pela necessidade de monitorar remotamente os seus astronautas ${ }^{(9)}$. Por volta de 1990, com o avanço da Internet e o advento dos computadores pessoais, os esforços e as possibilidades para a área aumentaram consideravelmente. Havia, porém, muitas dúvidas sobre o sistema e certa relutância dos profissionais da área sobre algo que ainda parecia novo e com eficácia e custo-benefício não comprovados ${ }^{(10)}$.
Há poucos estudos que comprovam o custo-benefício desta nova forma de comunicação na saúde. Há, porém, uma necessidade gigantesca de atender populações remotas, principalmente em países subdesenvolvidos e em desenvolvimento. Neste ponto, um interessante trabalho que pode ser citado é o desenvolvido no Estado do Pernambuco, região nordeste do Brasil, em que a Rede de Núcleos de Telessaúde (Rede NUTES), coordenada pela Universidade Federal de Pernambuco (UFPE), desenvolve atividades de telessaúde. O programa disponibiliza serviços de tele-educação, teleassistência e telegestão na rede pública de saúde do Estado, sendo prioridade às equipes de Saúde da Família(11).

O sistema de telemedicina no Brasil surgiu na década de 60 e consiste no uso da tecnologia para possibilitar cuidados à saúde nas situações em que a distância é um fator crítico $^{(12)}$.

O Estado de Minas Gerais conta, desde 2006, com um sistema de telecardiologia na Rede de Teleassistência de Minas Gerais (RTMG), que possui como um dos princípios norteadores, a implantação de telecardiologia a pequenos custos em cidades pequenas do interior do Brasil, reduzindo assim, encaminhamentos desnecessários, melhorando a qualidade da assistência e reduzindo o custo da atenção à saúde.

A RTMG já implantou os serviços de telemedicina em 780 municípios sendo que os mesmos estão interligados a sete polos universitários. A Universidade Federal de Minas Gerais (UFMG) foi denominada como polo coordenador do projeto, responsável assim pela articulação com os polos das demais instituições, Universidade Estadual de Montes Claros (UNIMONTES), Universidade Federal de Juiz de Fora (UFJF), Universidade Federal de Uberlândia (UFU), Universidade Federal do Triângulo Mineiro (UFTM), Universidade Federal de São João Del Rei (UFSJ) Campus Cento-Oeste "Dona Lindu" (CCO) localizado em Divinópolis e em 2016 foi incorporada a Universidade Federal dos Vales 
do Jequitinhonha e Mucuri (UFVJM). Deve-se ressaltar que o polo da UFVJM foi incorporado após a conclusão desta pesquisa.

Partindo do princípio que, doenças do aparelho circulatório estão entre as principais causas de mortalidade no Brasil e geram grandes custos ao orçamento do Ministério da Saúde (MS), principalmente na alta complexidade, é de extrema importância monitorar, planejar e intervir, reduzindo os índices de mortalidade e prevenindo agravos em saúde nessa área. Assim, questiona-se quais são as alterações eletrocardiográficas mais prevalentes?

Dessa forma, o objetivo deste estudo é analisar os eletrocardiogramas realizados pela Rede de Teleassistência de Minas Gerais no período de janeiro a dezembro de 2011, a fim de buscar a prevalência das alterações eletrocardiográficas.

\section{Método}

Foi realizado um estudo retrospectivo, exploratório e descritivo dos eletrocardiogramas no período de janeiro a dezembro de 2011 na RTMG. Neste período, a base de dados possui 318.387 exames laudados por cardiologistas, cobria 658 municípios e tinha 817 pontos de telessaúde, o que representava $77 \%$ de cobertura dos municípios do Estado de Minas Gerais.

O dimensionamento de uma amostra foi feito a partir da equação do intervalo de confiança para proporção.

O intervalo de confiança para proporção é: $\quad I C(p)=\hat{p} \pm Z_{\frac{\alpha}{2}} \sqrt{\frac{\hat{p} \hat{q}}{n}}$, em que $\hat{p}$ é a proporção amostral, $\hat{q}=1-\hat{p}$ e $Z_{\frac{\alpha}{2}}$ é o valor crítico que corresponde ao nível de confiança (1- $\alpha$ ) desejado(13). O termo $z_{\frac{\alpha}{2}} \sqrt{\frac{\hat{p} \hat{q}}{n}}$ é a margem de erro (E). Então, pode-se escrever que $Z_{\frac{\alpha}{2}} \sqrt{\frac{\hat{p} \widehat{q}}{n}} \leq E$. Logo, isolando o $n$, tem-se que:

$$
n \geq \frac{\left(z_{\alpha / 2}\right)^{2}}{(E)^{2}} \hat{p} \hat{q}
$$

Na prática, é razoável que o erro $\mathrm{E}$ a ser escolhido pelo usuário seja pequeno, por exemplo, $1 \%$ a $3 \%$. A margem de erro usada aqui foi de $1,6 \%$, ou seja, $E=0,016$. O nível de confiança foi de $95 \%(1-\alpha=0,95)$, logo $\alpha=$ 0,05 e $z_{0,05}=1,96$. Foi adotado $\hat{p}=\hat{q}=0,5$, pois assim, tem-se o valor máximo para $\hat{p} \hat{q}$, o qual garante o maior $n^{(13)}$.

Então, pode-se utilizar amostras de tamanho $n \geq 3752$. Assim sendo, o estudo contou com 4000 exames selecionados através da amostragem aleatória proporcional no período mencionado, o que garante que a amostra seja representativa. O número de eletrocardiogramas sorteados em cada polo foi proporcional à soma da população dos municípios que estão diretamente relacionados a cada polo. Foi utilizado o software Bioestat 5.0 para obtenção da amostra(14). $^{(14)}$

Para selecionar os exames da amostra e coletar as informações que interessam nesse estudo, foi desenvolvido um software utilizando o software Matlab ${ }^{(15)}$. Foram excluídos da amostra os exames que não possuíam laudo médico e aqueles que continham uma solicitação médica pedindo que o exame fosse realizado novamente devido a possíveis interferências e/ou troca de eletrodos durante a gravação do exame.

Conforme a Diretriz de Interpretação de Eletrocardiograma de Repouso da Sociedade Brasileira de Cardiologia - SBC(1), as alterações eletrocardiográficas podem ser agrupadas em seis categorias: arritmias; sobrecarga das câmeras cardíacas; bloqueios intraventriculares; isquemia, lesão e área eletricamente inativa; presença de marcapassos artificiais; e outras alterações. Os exames da amostra que apresentaram no laudo médico alguma alteração eletrocardiográfica foram agrupados para análise.

A pesquisa foi realizada após aprovação pelo Comitê de Ética em Pesquisa da Universidade Federal de Uberlândia (CEP/UFU), protocolo n. ${ }^{\circ}$ 029/12. Considerando o projeto Teleminas Saúde, um 
programa que durante o período do estudo abrangia 658 municípios em Minas Gerais e por se tratar de um estudo retrospectivo, foi dispensada pelo CEP a aplicação do Termo de Consentimento Livre e Esclarecido (TCLE) a todos os participantes indiretos da pesquisa.

Como não foi obtida a assinatura do TCLE dos participantes da pesquisa, foi respeitado o sigilo das informações e o anonimato, de acordo com a Resolução n.. 466/12 do Conselho Nacional de Saúde CNS $^{(16)}$. Deve-se ressaltar que os dados foram apresentados no seu conjunto.

\section{Resultados e Discussões}

A amostra, composta por 4000 exames, abrange a faixa etária de 0 a 101 anos, sendo 2414 (60,35\%) de pacientes do sexo feminino e 1586 (39,65\%) de pacientes do sexo masculino.

Deve-se ressaltar que esses laudos foram estabelecidos pelos cardiologistas do programa Teleminas Saúde e os eletrocardiogramas foram de realizados de forma não randomizada, ou seja, os exames são de pacientes que procuram as unidades de saúde credenciadas a RTMG e por alguma razão era solicitado pelo médico assistente da própria unidade a execução do exame.

Houve uma prevalência de 53,40\% de exames com laudo de eletrocardiograma dentro dos limites da normalidade e 40,23\% com algum tipo de alteração.

Apesar de constar na base de dados que todos os exames eram laudados, $13(0,33 \%)$ estavam sem laudo médico e 242 (6,05\%) continham uma solicitação médica pedindo que o exame fosse realizado novamente devido a possíveis interferências e/ou troca de eletrodos durante a gravação do exame.

Um estudo(17) realizado com ECG do mesmo programa da RTMG no ano de 2009, $n$ $=7709$ exames, concluiu que 56,87\% dos laudos eletrocardiográficos eram normais. Outra pesquisa ${ }^{(18)}$ com eletrocardiogramas também da RTMG, $n=290795$ exames, concluiu que $57,6 \%$ dos laudos eletrocardiográficos eram normais e ainda observou uma redução progressiva na prevalência de exames normais com aumento da idade.

Os exames da RTMG que possuíam no laudo médico "alterações eletrocardiográficas" foram agrupados de acordo com a alteração em seis polos, visto que o polo UFVJM foi incorporado somente em 2016.

A Tabela 1 e a Tabela 2 apresentam as alterações eletrocardiográficas e seus Intervalos de Confiança (IC) para cada polo da RTMG. A Tabela 3 apresenta as alterações eletrocardiográficas e seus IC para toda a RTMG.

Tabela 1. Prevalência das alterações eletrocardiográficas nos polos de Uberlândia, Uberaba e Divinópolis. Minas Gerais, Brasil, 2011.

\begin{tabular}{|c|c|c|c|c|c|c|}
\hline \multirow[b]{2}{*}{ Alteração } & \multicolumn{2}{|r|}{$\begin{array}{c}\text { Polo de } \\
\text { Uberlândia }\end{array}$} & \multicolumn{2}{|r|}{$\begin{array}{l}\text { Polo de } \\
\text { Uberaba }\end{array}$} & \multicolumn{2}{|r|}{$\begin{array}{l}\text { Polo de } \\
\text { Divinópolis }\end{array}$} \\
\hline & $n$ & $I C(p)^{*}$ & $n$ & $I C(p)^{*}$ & $n$ & $I C(p)^{*}$ \\
\hline Arritmias & 35 & $24,14 \pm 6,97$ & 27 & $18,24 \pm 6,22$ & 48 & $25,26 \pm 6,18$ \\
\hline Sobrecarga das câmeras cardíacas & 1 & $0,69 \pm 1,35$ & 7 & $4,73 \pm 3,42$ & 16 & $8,42 \pm 3,95$ \\
\hline Bloqueios intraventriculares & 37 & $25,52 \pm 7,10$ & 45 & $30,41 \pm 7,41$ & 45 & $23,68 \pm 6,05$ \\
\hline $\begin{array}{l}\text { Isquemia, lesão e área eletricamente } \\
\text { inativa }\end{array}$ & 3 & $2,07 \pm 2,32$ & 5 & $3,38 \pm 2,91$ & 4 & $2,11 \pm 2,04$ \\
\hline Presença de marcapassos artificiais & 2 & $1,38 \pm 1,90$ & 0 & $0 \pm 0$ & 1 & $0,53 \pm 1,03$ \\
\hline Outras alterações & 67 & $46,21 \pm 8,12$ & 64 & $43,24 \pm 7,98$ & 76 & $40,00 \pm 6,97$ \\
\hline
\end{tabular}

* $p \pm z \propto / 2 \sqrt{\frac{p(1-p)}{n}}(\%) \quad$ Fonte: dados da pesquisa. 
Tabela 2. Prevalência das alterações eletrocardiográficas nos polos de Juiz de Fora, Montes Claros e Belo Horizonte. Minas Gerais, Brasil, 2011.

\begin{tabular}{lcccccc}
\hline & \multicolumn{2}{c}{$\begin{array}{c}\text { Polo de } \\
\text { Juiz de Fora }\end{array}$} & \multicolumn{2}{c}{$\begin{array}{c}\text { Polo de } \\
\text { Montes Claros }\end{array}$} & \multicolumn{2}{c}{$\begin{array}{c}\text { Polo de } \\
\text { Belo Horizonte }\end{array}$} \\
\hline Alteração & $n$ & $\mathrm{IC}(\mathrm{p})^{*}$ & $n$ & $\mathrm{IC}(\mathrm{p})^{*}$ & $n$ & $\mathrm{IC}(\mathrm{p})^{*}$ \\
\hline Arritmias & 43 & $18,45 \pm 4,98$ & 103 & $23,62 \pm 3,99$ & 196 & $23,76 \pm 2,90$ \\
Sobrecarga das câmeras cardíacas & 13 & $5,58 \pm 2,95$ & 22 & $5,05 \pm 2,05$ & 49 & $5,94 \pm 1,61$ \\
Bloqueios intraventriculares & 68 & $29,18 \pm 5,84$ & 102 & $23,39 \pm 3,97$ & 210 & $25,45 \pm 2,97$ \\
Isquemia, lesão e área eletricamente & 11 & $4,72 \pm 2,72$ & 12 & $2,75 \pm 1,54$ & 42 & $5,09 \pm 1,50$ \\
inativa & 0 & $0 \pm 0$ & 4 & $0,92 \pm 0,89$ & 7 & $0,85 \pm 0,63$ \\
Presença de marcapassos artificiais & 98 & $42,06 \pm 6,34$ & 193 & $44,27 \pm 4,66$ & 321 & $38,91 \pm 3,33$ \\
Outras alterações & & & & & &
\end{tabular}

Fonte: dados da pesquisa

Tabela 3. Prevalência das alterações eletrocardiográficas em 2011 em todos os polos. Minas Gerais, Brasil, 2011.

\begin{tabular}{|c|c|c|c|}
\hline & \\
\hline & & \multicolumn{2}{|r|}{ Total } \\
\hline & Alteração & $n$ & $I C(p)^{*}$ \\
\hline & Arritmias & 452 & $22,86 \pm 1,85$ \\
\hline & Sobrecarga das câmeras cardíacas & 108 & $5,46 \pm 1,00$ \\
\hline & Bloqueios intraventriculares & 507 & $25,64 \pm 1,92$ \\
\hline & $\begin{array}{l}\text { Isquemia, lesão e área eletricamente } \\
\text { inativa }\end{array}$ & 77 & $3,89 \pm 0,85$ \\
\hline & Presença de marcapassos artificiais & 14 & $0,71 \pm 0,37$ \\
\hline & Outras alterações & 819 & $41,43 \pm 2,17$ \\
\hline
\end{tabular}

Fonte: dados da pesquisa

O maior índice de prevalência das alterações em todos os polos está relacionado aos bloqueios intraventriculares, ao todo, $25,64 \%$ dos exames alterados se enquadram nesta classificação.

O Bloqueio de Ramo Esquerdo (BRE) é um tipo de bloqueio intraventricular que foi encontrado em 55,82\% dos exames com bloqueios intraventriculares da RTMG. A prevalência aumenta com a idade, ocorrendo com pouca frequência em jovens saudáveis ${ }^{(19)}$.
O BRE, de forma usual, ocorre em pacientes com doença cardíaca prévia e pode estar associado com doença progressiva do sistema de condução(20). Aproximadamente, $30 \%$ dos pacientes com insuficiência cardíaca têm BRE, e em torno de $70 \%$ dos pacientes que desenvolvem $B R E$, tinham evidências prévias de sobrecarga ventricular esquerda no

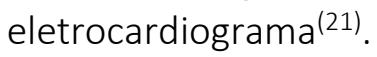

O BRE está associado com o risco aumentado de mortalidade cardiovascular por infarto e insuficiência cardíaca(20). 
O Bloqueio de Ramo Direito (BRD) também é um tipo de bloqueio intraventricular, sendo encontrado em $41.81 \%$ dos exames da pesquisa que apresentaram bloqueio intraventricular. Este tipo de bloqueio é um achado comum na população geral, sua prevalência também aumenta com a idade e ocorre em muitas pessoas sem evidência de doença cardíaca estrutural (19).

A elevada prevalência de BRD está associada à fragilidade do ramo direito(20). Algumas doenças podem causar BRD, como: cor pulmonale, embolia pulmonar, isquemia e infartos cardíacos, miocardite, hipertensão, cardiomiopatias e doença cardíaca congênita(19).

Há de se considerar que o Estado de Minas Gerais é considerado um dos estados brasileiros com maior prevalência da endemia chagásica e o BRD é o distúrbio de condução mais frequente deste tipo de cardiopatia.

A segunda alteração eletrocardiográfica mais prevalente em todos os polos foi às arritmias cardíacas, correspondendo a 22,86\% da população estudada.

As arritmias são alterações no ritmo cardíaco que, na maioria das vezes, ocorre de forma inesperada. As arritmias podem se originar na parte superior (átrios ou supraventriculares) ou inferior do coração (ventrículos). Dentre as arritmias supraventriculares, destacam-se: as extrassístoles atriais, taquicardia atrial, flutter e fibrilação atrial. A fibrilação atrial é bastante frequente na prática clínica. Trata-se de uma alteração no ritmo cardíaco, com contrações rápidas e não coordenadas dos átrios, que atinge boa parte da população na terceira idade. Nos ventrículos, a mais frequente é a extrassístole ${ }^{(22)}$.

Uma pesquisa ${ }^{(23)}$ realizada na cidade de São Paulo, revela que 21 mil pessoas são acometidas por morte súbita a cada ano, sendo 20\% em decorrência de problemas cardíacos. Estima-se que, no Brasil, cerca de 212 mil pessoas morram dessa causa por ano, 90\% delas por causa de arritmia cardíaca passível de ser tratada, se for diagnosticada a tempo.

A prevenção de arritmias envolve, além da prática de exercícios físicos e alimentação balanceada (baixa ingestão de sal e gorduras), a avaliação médica regular e o controle de fatores de risco de doenças, como: diabetes, obesidade, hipertensão e tabagismo (22).

\section{Conclusão}

A partir da amostragem de 4000 eletrocardiogramas provenientes da RTMG, os exames que possuíam no laudo médico alguma alteração eletrocardiográfica (40,23\%) foram agrupados de acordo com cada alteração. Desses, a principal alteração eletrocardiográfica encontrada em todos os polos foi relacionada aos bloqueios intraventriculares $(25,64 \%)$, sendo que o BRE está relacionado a $55,82 \%$ dos casos e o BRD a $41,81 \%$ dos casos. Geralmente, o BRE ocorre em pacientes com doença cardíaca prévia e a prevalência aumenta com a idade. O BRD também aumenta a prevalência com a idade e é um achado comum na população em geral.

$$
\text { As arritmias cardíacas (22,86\%) }
$$
correspondem à segunda alteração eletrocardiográfica mais prevalente em todos os polos. Posteriormente, encontram-se as alterações de sobrecarga das câmeras cardíacas (5,46\%); isquemia, lesão e área eletricamente inativa (3,89\%); e a presença de marcapassos artificiais $(0,71 \%)$.

Contudo, os resultados do estudo poderão fornecer subsídios que auxiliem no planejamento de políticas públicas, colaborando para nortear programas que promovam uma assistência de qualidade na área. Dessa forma, poderá contribuir para uma redução dos índices de mortalidade por doenças do aparelho circulatório e evitar a sobrecarga significativa no orçamento do MS na atenção da alta complexidade, através da prevenção e/ou controle de agravos em saúde. 


\section{Referências}

1. Pastore CA, Pinho JA, Pinho C, Samesima N, Pereira-Filho HG, Kruse JCL, et. al. III Diretrizes da Sociedade Brasileira de Cardiologia sobre análise e emissão de laudos eletrocardiográficos [Internet]. Sociedade Brasileira de Cardiologia: Arq Bras Cardiol; 2016 [citado em jan 10 2018]. Disponível em: http://publicacoes.cardiol.br/2014/diretrizes/ 2016/01_III_DIRETRIZES_ELETROCARDIOGR\% C3\%81FICOS.pdf .

2. Kawabata-Yoshihara LA, Benseñor IM, Kawabata VS, Menezes PR, Scazufca M, Lotufo PA. Prevalência de achados eletrocardiográficos no paciente idoso: estudo envelhecimento e saúde de São Paulo. Arq Bras Cardiol [Internet]. 2009 [citado em 10 jan 2018]; 93(6):651-56. Disponível em: http://www.scielo.br/pdf/abc/v93n6/15.pdf.

3. The top 10 causes of death worldwide [Internet]. World Health Organization: Geneva. 2015 [citado em 9 jul 2018]. Disponível em: www.who.int/mediacentre.

4. Ministério da Saúde (BR). Banco de dados de estatísticas vitais [Internet]. Departamento de Informática do SUS: Brasília. 2015 [citado em 9 jul 2018]. Disponível em: www.datasus.gov.br.

5. Tulu B, Chatterjee S, Maheshwari M. Telemedicine taxonomy: a classification tool. Telemed J E Health [Internet]. 2007 Jun [citado em 5 fev 2018]; 13(3):349-58. Disponível em: https://www.ncbi.nlm.nih.gov/pubmed/1760 3838.

6. Whitten $P$, Johannessen LK, Soerensen $T$, Gammon D, Mackert M. A systematic review of research methodology in telemedicine studies. J Telemed Telecare [Internet]. 2007 [citado em 7 fev 2018]; 13(5):230-5. Disponível em: https://www.ncbi.nlm.nih.gov/pubmed/1769 7509.
7. Artinian NT. Telehealth as a tool for enhancing care for patients with cardiovascular disease. I Cardiovasc Nurs [Internet]. 2007 Jan-Feb [citado em $20 \mathrm{fev}$ 2018]; 22(1):25-31. Disponível em: https://www.ncbi.n/m.nih.gov/pubmed/1722 4694.

8. Carvalho EM, Thiengo PCS, Gallach CH, Pérez Júnior EF, Toledo TT, Ferreira-Sae MCS. Cardiomiopatia não compactada: estudo de caso sob a ótica da sistematização da assistência de enfermagem. Rev Enferm Atual [Internet]. 2017 [citado em 10 jul 2018]; 82:120-27. Disponível em: https://revistaenfermagematual.com.br/uplo ads/revistas/20/revista.pdf.

9. Kim YS. Telemedicine in the U.S.A. with focus on clinical applications and issues. Yonsei Medical Journal [Internet]. 2004 Oct [citado em 20 fev 2018]; 45(5):761-75. Disponível em: https://www.ncbi.nlm.nih.gov/pubmed/1551 5185.

10. Bashshur RL, Shannon GW. History of telemedicine: evolution, context, and transformation. 1. ed. Nova York: Mary Ann Liebert; 2009.

11. Rede NUTES - Rede de Núcleos de Telessaúde [Internet]. Universidade Federal de Pernambuco. 2018 [citado em 10 jul 2018]. Disponível em: http://www.redenutes.ufpe.br.

12. Wen CL. Telemedicina e Telessaúde - um panorama no Brasil. Informática Pública [Internet]. 2008 [citado em 22 fev 2018]; 10(2):07-15. Disponível em: http://www.ip.pbh.gov.br/ANO10_N2_PDF/te lemedicina_telesaude.pdf.

13. Cochran WG. Sampling techniques. New York: John Wiley \& Sons; 1977.

14. Instituto de Desenvolvimento Sustentável Mamirauá. Software Bioestat [Internet]. Tefé: 
Instituto de Desenvolvimento Sustentável Mamirauá. [citado em 22 fev 2018]. Disponível em: https://www.mamiraua.org.br/pt$\mathrm{br} /$ downloads/programas/manual-dobioestat/

15. MathWorks. Software MATLAB [Internet] Massachusetts: MathWorks. [citado em $22 \mathrm{fev}$ 2018]. Disponível em: http://www.mathworks.com

16. Ministério da Saúde (BR). Resolução n.음 466, de 12 de Dezembro de 2012 [Internet]. Brasília. 2012 dez [citado em 11 jul 2018]. Disponível em: http://bvsms.saude.gov.br/bvs/saudelegis/cns /2013/res0466_12_12_2012.html.

17. Alkmin MBM, Abreu MP, Figueira RM, Cunha LR, Neme ES, Ribeiro ALP. Teleassistência para municípios remotos do Estado de Minas Gerais: ampliando a atuação geográfica dos hospitais universitários. Projeto Melhores Práticas [Internet]. 2010; [citado em 22 fev 2018]. Disponível em: http://melhorespraticas.mec.gov.br/arquivos/ ufmg_telemarketing.pdf.

18. Marcolino MS, Palhares D MF, Alkmim MBM, Ribeiro AL. Prevalence of normal electrocardiograms in primary care patients. Rev Assoc Med Bra [Internet]. 2014 [citado em 10 jan 2018]; 60(3):236-41. Disponível em: http://www.scielo.br/scielo.php?script=sci_ar ttext\&pid=S0104-42302014000300236.
19. Magalhães APA, Rodrigues HS. Significado clínico dos bloqueios de ramo e bloqueios fasciculares. Rev Sociedade Brasileira de Cardiologia do Estado do Rio Grande do Sul. 2011.

20. Braunwald E, Bonow R O, Mann D L, Zipes $D P$, Libby $P$, et al. Braunwald's heart disease. Philadelphia: Elsevier Saunders; 2011.

21. Imanishi R, Seto S, IChimaru S, NAkashima E, Yano K, Akahoshi M. Prognostic significance of incident complete left branch block observed over a 40-year period. Am J Cardiol [Internet]. 2006 [citado em 10 jan 2018], 98:644-8. Disponível em: https://www.ncbi.nlm.nih.gov/pubmed/1692 3453.

22. Campos MV. Mortes súbitas - 90\% delas por causa de arritmia cardíaca passível de ser tratada. Instituto do Coração do Hospital de Clínicas [Internet]. 2010 [citado em 5 dez 2017] Disponível em: http://www.hcor.com.br/especialidadesservicos/especialidades/cardiologia/arritmia/.

23. Cintra F. Arritmias [Internet]. 2012 [citado em 5 dez 2017]. Disponível em: https://www.einstein.br/especialidades/cardi ologia/doencas-sintomas/arritmias. 